\title{
Immunomodulatory effect of captopril and local irradiation on myeloid-derived suppressor cells
}

\author{
Won Kyung Cho, MD¹, Sung-Won Shin, PhD', Shin-Yeong Kim, MS', Chang-Won Hong, MD, PhD², \\ Changhoon Choi, PhD', Won Park, MD, PhD', Jae Myoung Noh, MD \\ 'Department of Radiation Oncology, Samsung Medical Center, Sungkyunkwan University School of Medicine, Seoul; \\ ${ }^{2}$ Department of Physiology, Kyungpook National University School of Medicine, Daegu, Korea
}

\begin{abstract}
Purpose: This study is to investigate the effect of captopril when combined with irradiation.
Materials and Methods: 4T1 (mouse mammary carcinoma) cells were injected in the right hind leg of Balb/c mice. Mice were randomized to four groups; control (group 1), captopril-treated (group 2), irradiated (group 3), irradiated and captopril-treated concurrently (group 4). Captopril was administered by intraperitoneal injection $(10 \mathrm{mg} / \mathrm{kg}$ ) daily and irradiation was delivered on the tumor-bearing leg for 15 Gy in 3 fractions. Surface markers of splenic neutrophils (G-MDSCs) and intratumoral neutrophils (tumorassociated neutrophils [TANs]) were assessed using flow cytometry and expression of vascular endothelial growth factor (VEGF) and hypoxia-inducible factor 1 alpha (HIF-1 $\alpha$ ) of tumor was evaluated by immunohistochemical (IHC) staining.

Results: The mean tumor volumes ( \pm standard error) at the 15 th day after randomization were 1,382.0 $( \pm 201.2) \mathrm{mm}^{3}$ (group 1), $559.9( \pm 67.8) \mathrm{mm}^{3}$ (group 3), and $370.5( \pm 48.1) \mathrm{mm}^{3}$ (group 4), respectively. For G-MDSCs, irradiation reversed decreased expression of CD101 from tumor-bearing mice, and additional increase of CD101 expression was induced by captopril administration. Similar tendency was observed in TANs. The expression of tumor-necrosis factor-associated molecules, CD120 and CD137, are increased by irradiation in both G-MDSCs and TANs. Further increment was observed by captopril except CD120 in TANs. For IHC staining, VEGF and HIF-1 $\alpha$ positivity in tumor cells were decreased when treated with captopril.

Conclusion: Captopril is suggested to have additional effect when combined to irradiation in a murine tumor model by modulation of MDSCs and angiogenesis.
\end{abstract}

Keywords: Radiotherapy, Angiotensin converting enzyme inhibitors, Neutrophil

\section{Introduction}

Neutrophils are essential for host defense against invading pathogens [1]. Myeloid-derived suppressor cells (MDSCs) are composed of monocytic (Ly6C+) and granulocytic (Ly6G+) lineages, and associated with tumor escape from immune regulation $[2,3]$. It differentiate to mature macrophages (tumor-associated macrophage [TAM]) and neutrophils (tumor-associated neutrophil [TAN]), respectively [4]. TAN can polarize to either hypersegmented anti-tumor N1 TAN or protumor N2 TAN, and the potential role of TGF-beta blockade in the recruitment and activation of TAN with an anti-tumor phenotype has been suggested [4]. Several clinical studies demonstrated poor prognosis of intratumoral neutrophils,

Received 2 June 2016, Revised 10 July 2016, Accepted 12 July 2016.

Correspondence: Jae Myoung Noh, MD, Department of Radiation Oncology, Samsung Medical Center, Sungkyunkwan University School of Medicine, 81 Irwon-ro, Gangnam-gu, Seoul 06351, Korea. Tel: +82-2-3410-2612, Fax: +82-2-3410-2619, E-mail: rodrno@skku.edu

(C) This is an Open Access article distributed under the terms of the Creative Commons Attribution Non-Commercial License (http://creativecommons.org/ licenses/by-nc/4.0/) which permits unrestricted non-commercial use, distribution, and reproduction in any medium, provided the original work is properly cited.

www.e-roj.org 
probably pro-tumor N2 TAN, in several human cancers such as renal cell carcinoma, non-small cell lung cancer, hepatocellular carcinoma, and cervical cancer [5-8]. Based on these backgrounds, several therapeutic strategies targeting MDSCs or TANs as cancer therapy have been suggested [9-11]. Angiotensin converting enzyme inhibitors (ACEis), which have been demonstrated to induce neutrophil hypersegmentation [12], is one of these strategies. Attenuation of tumor growth by ACEis via polarization of neutrophils toward antitumor phenotype was recently reported [13].

Radiation therapy (RT) is a widely used local treatment modality for cancer. But, local ineffectiveness of RT has still existed and MDSCs are suggested to be one of the key components of radioresistance $[14,15]$. Therefore, ACEis could be a potential radiosensitizing strategy via modulating MDSCs. In terms of radiation oncology, S-nitrosocaptopril was demonstrated to have the potential of radiosensitizing effect through improving tumor oxygenation [16]. But the radiosensitizing effect of renin-angiotensin system (RAS) blockade has not been widely studied, while decreased risk of radiation pneumonitis by ACEis has been relatively well investigated $[17,18]$.

The purpose of this study was to investigate the effect of captopril when combined with irradiation through modulation of MDSCs and tumor angiogenesis.

\section{Materials and Methods}

\section{Animals \&t tumors}

The animal experiments were reviewed and approved by the Institutional Animal Care and Use Committee (IACUC; 20140212001) of Samsung Biomedical Research Institute. A 7-week-old female Balb/c mice were purchased from Orient Bio (Gapyeong, Korea). 4T1 (mouse mammary carcinoma) cells were purchased from American Type Culture Collection (Manassas, VA, USA). Cells were cultured in Dulbecco's Modified Eagle's medium (Gibco, Carlsbad, CA, USA) supplemented with 10\% fetal bovine serum (Gibco), and $1 \times 10^{5}$ cells were injected in the right hind leg. Tumor volumes were measured every 3 days with calipers and calculated according to following formula:

$$
\text { Volume }=\mathrm{D}_{\text {Short }}^{2} \times \mathrm{D}_{\text {Long }} \div 2
$$

When the mean tumor volume reached $120 \mathrm{~mm}^{3}$, mice were randomized to four groups (group 1, control; group 2, captopril-treated; group 3, irradiated; group 4, irradiation and captopril-treated mice).

\section{Drug treatment $\mathbb{C}$ irradiation}

Captopril was purchased from Sigma-Aldrich (St Louis, MO, USA). It was administered by intraperitoneal injection (10 mg/ $\mathrm{kg}$ ) daily since the day of randomization. The injection was continued until the day before sacrifice. From the 3rd day of drug treatment, irradiation was delivered on the tumor-bearing right hind leg for 3 consecutive days at a dose of 5 Gy per fraction to a total dose of $15 \mathrm{~Gy}$. We used 4-MV photon beams generated by linear accelerator (Varian Medical Systems, Palo Alto, CA, USA). During the irradiation, mice were anesthetized by intraperitoneal injection of tiletamine + zolazepam (50 mg/ $\mathrm{kg}$ ) and xylazine (10 $\mathrm{mg} / \mathrm{kg})$ under prescription of veterinarian.

\section{Fluorescence-activated cell sorting (FACS) analysis}

When tumors reached $1,000 \mathrm{~mm}^{3}$ in volume, we sacrificed the mice and harvested the tumors and spleens. After measuring weights of tumors and spleens, tumors were minced with MACS dissociator (Miltenyi Biotec, Bergisch Gladbach, Germany) and further digested with $2 \mathrm{mg} / \mathrm{mL}$ DNase I (SigmaAldrich) and $4 \mathrm{mg} / \mathrm{mL}$ collagenase type IV (Sigma-Aldrich) for an 1 hour. Spleens were grounded with slide glass. Cells were further fixed with Cytofix (BD biosciences, San Jose, CA, USA) and resuspended in staining buffer (BD biosciences). After gating based on forward/side scatter profiles, neutrophils were further gated by Ly6G (FITC, 551460; PE, 551461; BD bioscience) and CD11b (PerCP, 550993; BD bioscience) expression. Following neutrophil phenotypic markers were examined: cell adhesion-associated molecules, CD43 (PE, 120431-81; eBioscience, San Diego, CA, USA), CD98 (PE, 12 0981-81; eBioscience), CD101 (PE, 12-1011-80, eBioscience); tumor necrosis factor (TNF)-associated molecules, CD120 (PE, 550088; BD bioscience), CD137 (PE, 558976; BD bioscience), CD256 (PE, 136703; BD bioscience). Flow cytometry and data analysis was performed with BD FACS Calibur and FlowJo software (TreeStar Inc., Ashland, OR, USA), respectively.

\section{Immunohistochemical (IHC) staining}

Tumor tissues were prepared to formalin-fixed, paraffinembedded 4- $\mu \mathrm{m}$ thick sections. The sections were deparaffinized in xylene, rehydrated in graded alcohol, and transferred to $0.01 \mathrm{M}$ phosphate-buffered saline (PBS, pH 7.4). After heat induced epitope retrieval (HIER) with citrate buffer ( $\mathrm{pH}$ 6.0; Dako, Carpinteria, CA, USA) for 3 minutes at $121^{\circ} \mathrm{C}$ to reveal hidden antigen epitopes, endogenous peroxidase was blocked with $3 \%$ hydrogen peroxide in PBS for 10 minutes 
at room temperature. After washing in PBS buffer, sections were treated with serum free blocking solution (Dako) for 20 minutes at room temperature to block nonspecific binding. Subsequently, sections were incubated with anti-vascular endothelial growth factor (VEGF) rabbit polyclonal antibody (5 $\mathrm{\mu g} / \mathrm{mL} ;$ Abcam, Cambridge, UK) and anti-hypoxia-inducible factor 1 alpha (anti-HIF-1 $\alpha$ ) mouse monoclonal antibody (1/50; Novus, Littleton, CO, USA) overnight at $4^{\circ} \mathrm{C}$. After washing in PBS, the sections were incubated for 30 minutes at room temperature with HRP-labeled polymer conjugated secondary antibodies against rabbit immunoglobulin G (VEGF; Dako) or mouse immunoglobulin G (HIF-1 $\alpha$; Dako). The color reaction was developed using the ready-to-use 3,3'-diaminobenzidine (DAB) substrate-chromogen solution (Dako) for 5 minutes and then washed with distilled water. Finally, sections were lightly counterstained with Mayer's hematoxylin for 30 seconds before dehydration and mounting.

\section{Statistical analysis}

Relative expression level of HIF-1 $\alpha$ and VEGF between groups was calculated by positive pixel count using algorithm Positive Pixel Count version 9 of Image Scope software (Aperio Technologies, Vista, CA, USA). Tumor volume and weight of spleen and tumor between the groups were compared by Mann-Whitney $U$ test. A p-value of 0.05 or less was considered statistically significant. The descriptive analysis, graphing of the experiment results were conducted using GraphPad prism 6.0 software (GraphPad Software Inc., San Diego, CA, USA).

\section{Results}

\section{In vivo tumor growth inhibition of irradiation}

Fig. 1 shows tumor growth delay by irradiation with or without captopril treatment. At the 22nd day after tumor cell injection, which was 15 days after randomization, mean tumor volume ( \pm standard error) of group 1 (control) was 1,382.0 $( \pm 201.2)$ $\mathrm{mm}^{3}$. Irradiation alone (group 3) attenuated it to $559.9( \pm 67.8)$ $\mathrm{mm}^{3}(\mathrm{p}=0.0007)$, and combined captopril treatment (group 4) further decreased tumor growth to $370.5( \pm 48.1) \mathrm{mm}^{3}(p=$ 0.0007). Tumor weight according to irradiation and/or captopril treatment demonstrated similar results (Fig. 2A). The mean tumor weights ( \pm standard error) were $1,416( \pm 107.8) \mathrm{mg}$ in group 1 and they were decreased in group 3 to $590.3( \pm 37.58)$ $\mathrm{mg}(\mathrm{p}<0.0001)$, and further reduced to $398.4( \pm 69.9) \mathrm{mg}$ in group 4 ( $p=0.0007$ ). Interestingly, local RT also attenuated tumor-induced splenomegaly (Fig. 2B). The mean spleen weights ( \pm standard error) were $719.1( \pm 87.22) \mathrm{mg}$ in group

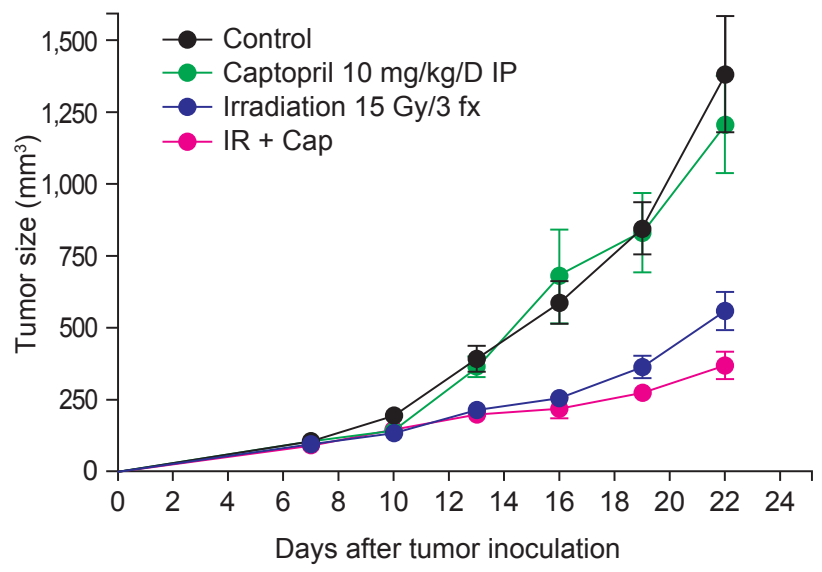

Fig. 1. Growth curves for tumors in control, captopril-treated, irradiated, and irradiation plus captopril-treated (IR + Cap) mice ( $n$ $=5-10)$. All results are shown as mean \pm standard error of mean. IP, Intraperitoneal injection.
A

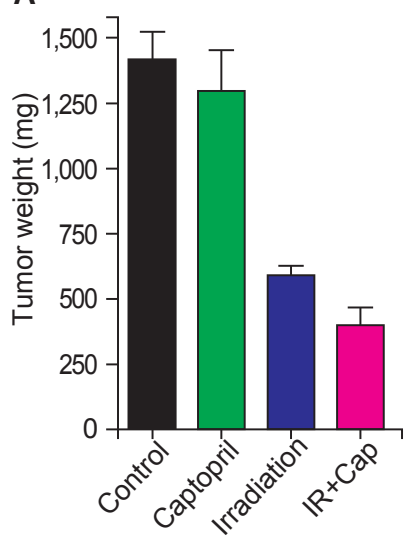

B

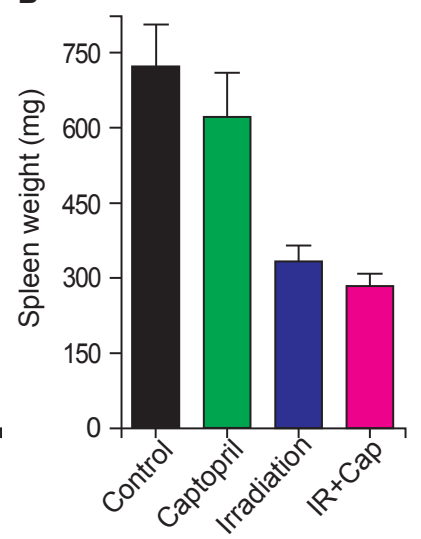

Fig. 2. (A) Tumor weight and (B) spleen weight in control, captopril-treated, irradiated, and irradiation plus captopril-treated $(I R+$ Cap $)$ mice $(n=5-10)$. All results are shown as mean \pm standard error of mean.

1 and $330.9( \pm 33.44) \mathrm{mg}$ in group $3(p=0.0052)$, and 280.7 $( \pm 28.32) \mathrm{mg}$ in group $4(\mathrm{p}=0.0006)$.

\section{Expression of neutrophil surface markers in spleen and tumor}

Fig. 3 demonstrates the results of flow cytometry for several phenotypic markers for neutrophil of spleen and tumor, representing G-MDSCs and TANs respectively. In G-MDSCs from tumor-bearing mice, expression of CD43 and CD98 tend to increase by irradiation. Irradiation numerically reversed decreased expression of CD101 in G-MDSCs from tumorbearing mice, and additional increase of CD101 expression 
was induced by captopril administration. Similar tendency was observed in TANs. Expression of TNF-associated molecules, CD120 and CD137, was increased by irradiation in both G-MDSCs and TANs. Further increment was observed by captopril except CD120 in TANs. The changes mentioned above were not statistically significant. Another TNF-associated molecule CD256 was decreased by irradiation in G-MDSCs and it was reversed by captopril, which was the only significant change $(p=0.0266)$.

\section{Expression of vascular endothelial growth factor and hypoxia-inducible factor $-1 \alpha$}

Fig. 4 demonstrates the results of IHC staining for VEGF and HIF- $1 \alpha$ in tumor tissues. VEGF and HIF- $1 \alpha$ expression decreased in captopril-treated groups. VEGF and HIF-1 $\alpha$ expression increased in irradiated tumor tissues compared to control group and it was reversed by concurrent captopril treatment.

\section{Discussion and Conclusion}

In this study, we tried to investigate the effect of ACEi,
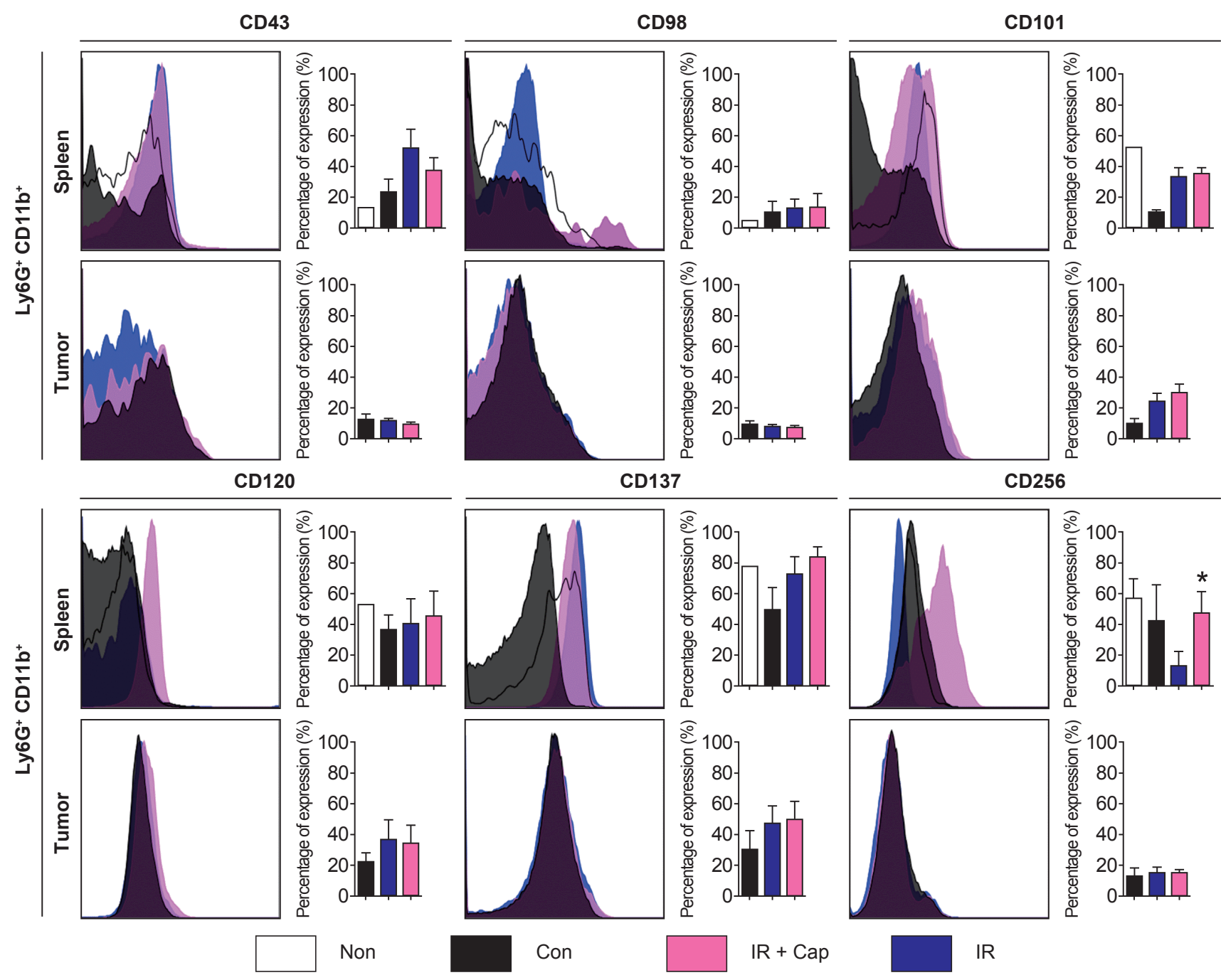

Fig. 3. Phenotypic markers of splenic and intratumoral neutrophils (left, the representative flow cytometry histogram for each phenotypic marker; right, quantification of each phenotypic marker's expression. All results are shown as mean \pm standard error of mean. All the $p$-values between control and irradiated mice or between irradiated and irradiation plus captopril-treated mice were not reported if it was not significant $(p \geq 0.05)$. Non, mice without tumors; Con, control mice with tumor; IR + Cap, irradiation plus captopril-treated mice; IR, irradiated mice. $n=5-10$ mice per each group; ${ }^{*} p<0.05$ compared to irradiated mice. 
A

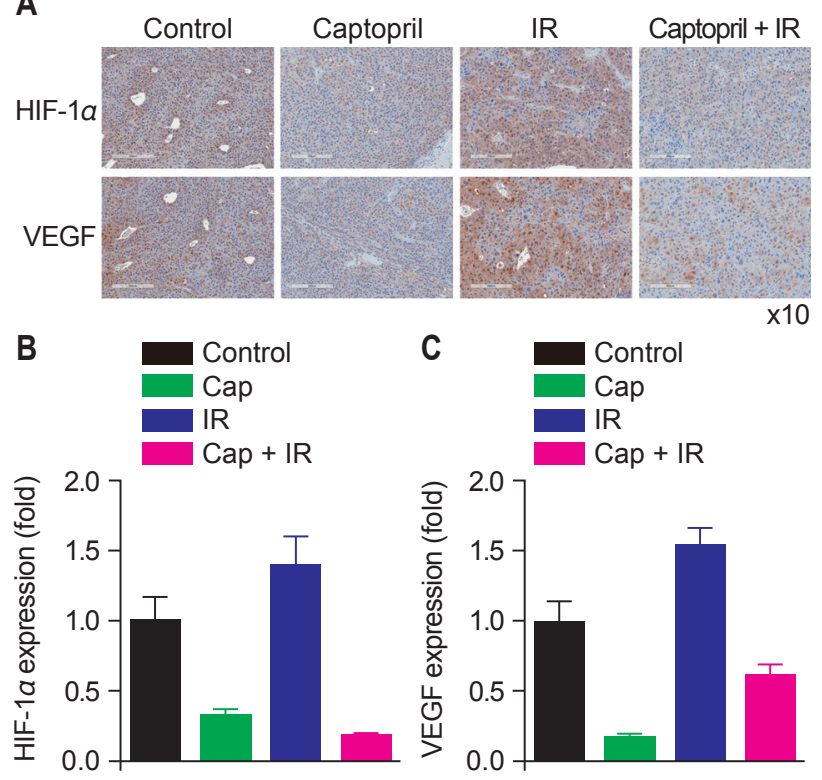

Fig. 4. Expression of vascular endothelial growth factor (VEGF) and hypoxia-inducible factor 1 alpha (HIF-1 $\alpha$ ) decreased in tumor cells when captopril was given concurrently with radiation compared to untreated, irradiated, or captopril-treated tumors. (A) Immunohistochemistry (IHC) staining (brown) of VEGF and HIF$1 \alpha$ in tumor cells of untreated mice (Control), irradiated mice (IR), captopril-treated (Cap) and radiation plus captopril-treated mice $(\mathrm{Cap}+\mathrm{IR})(\mathrm{HEE}, \times 10)$. Relative expression of HIF-1 $\alpha$ (B) and VEGF (C) in treated groups compared to control group.

captopril when combined with irradiation through MDSC modulation. The RAS influences tumor growth, in addition to its systemic actions on cardiovascular homeostasis [19]. Angiotensin II, which is the main effector of the RAS, stimulates the expression of VEGF, basic fibroblast growth factor (b-FGF), and platelet-derived growth factor (PDGF) via AT1 receptor (AT1R) [20-22]. There have been several studies that showed decreased tumor volume and decreased metastases by RAS blockade in vivo [19]. Modulation of angiogenesis, cellular proliferation, immune response, and extracellular matrix formation are suggested as underlying mechanisms. Furthermore, a clinical study showed that clinical outcomes in patients with advanced pancreatic cancer were improved by the combination of RAS blockade and gemcitabine [23], while the subsequent phase II trial failed to demonstrate activity of gemcitabine and candesartan against advanced pancreatic cancer [24].

MDSCs are one of the key components of immune evasion of cancer [10]. Increased MDSC frequencies were observed in cervical cancer patients with tumor-related leukocytosis
(TRL), which was associated with larger tumor size, advanced clinical stage, and shorter overall survival [15]. Tumor-derived granulocyte colony-stimulating factor (G-CSF) was a possible mediator of TRL and G-CSF-producing cervical cancer cell line grew significantly faster and was less sensitive to RT. Removal of spleen, which is a supplier of MDSCs, and antigranulocyte receptor $1(\mathrm{Gr}-1)$ neutralizing antibody resulted in a decrease of G-CSF-derived tumor burden and an increase of radiosensitivity. This suggests that therapeutic approaches targeting G-CSF-induced MDSCs might be valuable as a radiosensitizing strategy in patients with TRL [15].

Recently, Shrestha et al. [13] demonstrated neutrophil hypersegmentation induced by ACEis through mechanistic target of rapamycin (mTOR) pathway. In a murine model, ACEis attenuated tumor growth and inhibited tumor-induced polarization of neutrophils to a protumoral phenotype. Based on these findings, we investigated the effect of RT with or without captopril on expression of surface markers of MDSCs and TANs. Captopril showed certain degree of additional tumor growth delay in this study, and some surface markers were affected by administration of captopril addition to RT. Cell adhesion-associated molecules (CD43, CD98, CD101) and TNFassociated molecules (CD120, CD137, CD256) were influenced by irradiation.

CD43 is expressed in tumor-infiltrating neutrophils [25]. It has been known to have adhesive role in neutrophil recruitment [26]. The expression of CD43 in G-MDSCs of tumor-bearing mice was significantly increased, and it was further increased by irradiation (Fig. 3). Given that integrin and selecting is involved in MDSC trafficking [27], irradiation seems to be associated with neutrophil trafficking. CD98 is a large amino acid transporter that has important role in mTOR signaling, and known to be an adverse prognostic biomarker in cancer [28-30]. It establishes a positive loop that amplifies in mTOR signaling in T cells $[31,32]$. Through mTOR signaling, CD98 seems to mediate prolonged survival of G-MDSCs in tumor-bearing mice. Increased expression of CD98 in tumor-bearing mice was observed in this study, and irradiation decreased expression of CD98 in TANs, although it was not observed in MDSCs. CD101 is an immunoglobulin superfamily member 2 which also involves in neutrophil trafficking [33]. Decreased expression of CD101 in G-MDSCs from tumor-bearing mice and reversal by irradiation suggests the modulatory effect of RT on dysregulation of neutrophil trafficking.

CD120 is a TNF receptor that found in hematopoietic cells [34]. Irradiation increased expression of CD120 in G-MDSCs 
and TANs, suggesting induction of apoptosis. CD137 is another TNF receptor that have been shown to have a pro-apoptotic function in granulocytes [35]. Similar to CD120, its expression was increased by irradiation, and further increment was induced by captopril administration. CD256, a proliferationinducing ligand (APRIL) is a TNF family cytokine that promotes tumor growth [36]. Reduction of CD256 expression by irradiation was demonstrated in G-MDSCs and it was reversed by captopril administration.

We also examined IHC staining for VEGF and HIF-1 $\alpha$. VEGF has been the subject of intense clinical interest with many clinical trials targeting VEGF or VEGF signaling [19]. The RAS contributed to cellular proliferation and tumor-associated fibrosis and blockade of RAS might be beneficial in terms of anti-VEGF strategies. Our results meet the anti-VEGF effect of captopril, when combined to RT. HIF- $1 \alpha$ is a transcription factors that promotes tumor growth and metastasis through initiating angiogenesis via hypoxia-induced VEGF expression pathways and regulating energy metabolism to survive cellular hypoxia $[37,38]$. Along with its association with poor prognosis and resistance to RT, HIF- $1 \alpha$ could be another drug target of cancer treatment [37-39]. And decreased expression of HIF$1 \alpha$ on IHC staining of our study implicated the potential antitumor activity of RAS blockade through modulating angiogenesis.

Interestingly, local RT also attenuated tumor-induced splenomegaly (Fig. 2B). Tumor-induced splenomegaly and attenuation by captopril was demonstrated by previous study [13]. In addition, we found attenuation of splenomegaly by local RT on tumor-bearing leg, while spleen was not included in the RT field. Immunoadjuvant effect of local RT has been recently demonstrated $[40,41]$. Increased antigenic expression, bridging innate and adaptive immunity, promoting tumor recognition by preexisting tumor-specific $T$ cells, and induction of tumor-specific cytotoxic T cells have been suggested as the roles of RT in induction of the antitumor immune response [42]. While previous studies focused on adaptive immune system based on T cell immunity, our study focused on innate immune system based on granulocytic-lineage.

Taken together, captopril modulated neutrophil trafficking and reduced angiogenesis. Combined treatment with captopril and irradiation increased tumor cell killing compared to RT or captopril alone treatment. The effect of captopril and irradiation was observed not only in TANs, but also in G-MDSCs in spleen which was not included in irradiation field. It suggests that local RT can have systemic immunoediting effect on MDSCs. However, we need more data to confirm the captopril as a radiosensitizer and further studies to investigate the mechanism are warranted.

\section{Conflict of Interest}

No potential conflict of interest relevant to this article was reported.

\section{Acknowledgments}

This work was supported by the Korean Society for Radiation Oncology (KOSRO) Young Investigator Fund (2013).

\section{References}

1. Mantovani A, Cassatella MA, Costantini C, Jaillon S. Neutrophils in the activation and regulation of innate and adaptive immunity. Nat Rev Immunol 2011;11:519-31.

2. Schreiber RD, Old LJ, Smyth MJ. Cancer immunoediting: integrating immunity's roles in cancer suppression and promotion. Science 2011;331:1565-70.

3. Dunn GP, Bruce AT, Ikeda H, Old LJ, Schreiber RD. Cancer immunoediting: from immunosurveillance to tumor escape. Nat Immunol 2002;3:991-8.

4. Fridlender ZG, Sun J, Kim S, et al. Polarization of tumorassociated neutrophil phenotype by TGF-beta: "N1" versus "N2" TAN. Cancer Cell 2009;16:183-94.

5. Carus A, Ladekarl M, Hager $H$, Nedergaard BS, Donskov F. Tumour-associated CD66b + neutrophil count is an independent prognostic factor for recurrence in localised cervical cancer. Br J Cancer 2013;108:2116-22.

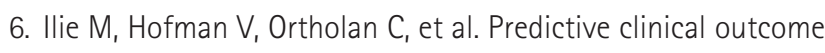
of the intratumoral CD66b-positive neutrophil-to-CD8positive T-cell ratio in patients with resectable nonsmall cell lung cancer. Cancer 2012;118:1726-37.

7. Li YW, Qiu SJ, Fan J, et al. Intratumoral neutrophils: a poor prognostic factor for hepatocellular carcinoma following resection. J Hepatol 2011;54:497-505.

8. Jensen HK, Donskov F, Marcussen N, Nordsmark M, Lundbeck $F$, von der Maase $H$. Presence of intratumoral neutrophils is an independent prognostic factor in localized renal cell carcinoma. J Clin Oncol 2009;27:4709-17.

9. Brandau S, Dumitru CA, Lang S. Protumor and antitumor functions of neutrophil granulocytes. Semin Immunopathol 2013;35:163-76.

10. Diaz-Montero CM, Finke J, Montero AJ. Myeloid-derived suppressor cells in cancer: therapeutic, predictive, and prognostic implications. Semin Oncol 2014;41:174-84.

11. Gregory AD, Houghton AM. Tumor-associated neutrophils: new targets for cancer therapy. Cancer Res 2011;71:2411-6. 
12. Okutan V, Kurekci AE, Sarici SU, et al. Neutrophil hypersegmentation in children receiving angiotensin converting enzyme inhibitors. Turk J Pediatr 2008;50:438-42.

13. Shrestha S, Noh JM, Kim SY, et al. Angiotensin converting enzyme inhibitors and angiotensin $I$ receptor antagonist attenuate tumor growth via polarization of neutrophils toward an antitumor phenotype. Oncoimmunology 2015;5:e1067744.

14. Gabrilovich DI, Nagaraj S. Myeloid-derived suppressor cells as regulators of the immune system. Nat Rev Immunol 2009;9:162-74.

15. Mabuchi S, Matsumoto $Y$, Kawano M, et al. Uterine cervical cancer displaying tumor-related leukocytosis: a distinct clinical entity with radioresistant feature. J Natl Cancer Inst 2014;106:dju147.

16. Jordan BF, Peeterbroeck J, Karroum 0, et al. Captopril and S-nitrosocaptopril as potent radiosensitizers: comparative study and underlying mechanisms. Cancer Lett 2010;293:213-9.

17. Ghosh SN, Zhang R, Fish BL, et al. Renin-angiotensin system suppression mitigates experimental radiation pneumonitis. Int J Radiat Oncol Biol Phys 2009;75:1528-36.

18. Kharofa J, Cohen EP, Tomic R, Xiang Q, Gore E. Decreased risk of radiation pneumonitis with incidental concurrent use of angiotensin-converting enzyme inhibitors and thoracic radiation therapy. Int J Radiat Oncol Biol Phys 2012;84:23843.

19. Ager El, Neo J, Christophi C. The renin-angiotensin system and malignancy. Carcinogenesis 2008;29:1675-84.

20. Fujita M, Hayashi I, Yamashina S, Itoman M, Majima M. Blockade of angiotensin AT1a receptor signaling reduces tumor growth, angiogenesis, and metastasis. Biochem Biophys Res Commun 2002;294:441-7.

21. Wysocki PJ, Kwiatkowska EP, Kazimierczak U, Suchorska W, Kowalczyk DW, Mackiewicz A. Captopril, an angiotensinconverting enzyme inhibitor, promotes growth of immunogenic tumors in mice. Clin Cancer Res 2006;12:4095-102.

22. Huang $W$, Wu YL, Zhong J, Jiang FX, Tian XL, Yu LF. Angiotensin II type 1 receptor antagonist suppress angiogenesis and growth of gastric cancer xenografts. Dig Dis Sci 2008;53:1206-10.

23. Nakai $Y$, Isayama $H$, ljichi $H$, et al. Inhibition of reninangiotensin system affects prognosis of advanced pancreatic cancer receiving gemcitabine. Br J Cancer 2010;103:1644-8.

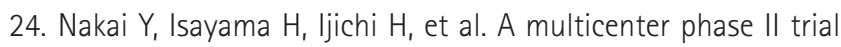
of gemcitabine and candesartan combination therapy in patients with advanced pancreatic cancer: GECA2. Invest New Drugs 2013;31:1294-9.

25. Virtuoso $L P$, Harden $J$, Sotomayor $P$, et al. Characterization of iNOS(+) Neutrophil-like ring cell in tumor-bearing mice. J Transl Med 2012;10:152.
26. Moreno-Amaral AN, Gout E, Danella-Polli C, et al. M-ficolin and leukosialin (CD43): new partners in neutrophil adhesion. J Leukoc Biol 2012;91:469-74.

27. Talmadge JE, Gabrilovich DI. History of myeloid-derived suppressor cells. Nat Rev Cancer 2013;13:739-52.

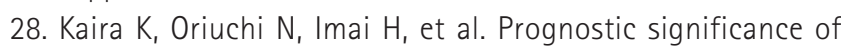
L-type amino acid transporter 1 expression in resectable stage I-III nonsmall cell lung cancer. Br J Cancer 2008;98:742-8.

29. Cantor JM, Ginsberg MH. CD98 at the crossroads of adaptive immunity and cancer. J Cell Sci 2012;125(Pt 6):1373-82.

30. Nguyen HT, Dalmasso G, Torkvist L, et al. CD98 expression modulates intestinal homeostasis, inflammation, and colitisassociated cancer in mice. J Clin Invest 2011;121:1733-47.

31. Nicklin $P$, Bergman $P$, Zhang $B$, et al. Bidirectional transport of amino acids regulates $m T O R$ and autophagy. Cell 2009;136:521-34.

32. Chi H. Regulation and function of mTOR signalling in T cell fate decisions. Nat Rev Immunol 2012;12:325-38.

33. Mohammed JP, Fusakio ME, Rainbow DB, et al. Identification of CD101 as a susceptibility gene for Novosphingobium aromaticivorans-induced liver autoimmunity. J Immunol 2011;187:337-49.

34. Balkwill F. Tumour necrosis factor and cancer. Nat Rev Cancer 2009;9:361-71.

35. Simon HU. Evidence for a pro-apoptotic function of CD137 in granulocytes. Swiss Med Wkly 2001;131:455-8.

36. Garcia-Castro A, Zonca M, Florindo-Pinheiro D, et al. APRIL promotes breast tumor growth and metastasis and is associated with aggressive basal breast cancer. Carcinogenesis 2015;36:574-84.

37. Powis G, Kirkpatrick L. Hypoxia inducible factor-1alpha as a cancer drug target. Mol Cancer Ther 2004;3:647-54.

38. Bos R, van der Groep P, Greijer AE, et al. Levels of hypoxiainducible factor-1alpha independently predict prognosis in patients with lymph node negative breast carcinoma. Cancer 2003;97:1573-81.

39. Aebersold DM, Burri P, Beer KT, et al. Expression of hypoxiainducible factor-1alpha: a novel predictive and prognostic parameter in the radiotherapy of oropharyngeal cancer. Cancer Res 2001;61:2911-6.

40. Postow MA, Callahan MK, Barker CA, et al. Immunologic correlates of the abscopal effect in a patient with melanoma. N Engl J Med 2012;366:925-31.

41. Stamell EF, Wolchok JD, Gnjatic S, Lee NY, Brownell I. The abscopal effect associated with a systemic anti-melanoma immune response. Int J Radiat Oncol Biol Phys 2013;85:293-5.

42. Kalbasi $A$, June $C H$, Haas $N$, Vapiwala $N$. Radiation and immunotherapy: a synergistic combination. J Clin Invest 2013;123:2756-63. 